\title{
The causal role of smoking on the risk of headache. A Mendelian randomization analysis
}

\section{in the HUNT Study}

Marianne Bakke Johnsen, $\mathrm{PhD}^{1,2,3 *}$, Bendik Slagsvold Winsvold, $\mathrm{PhD}^{2,4}$, Sigrid Børte, $\mathrm{MD}^{1,2}$, Gunnhild Åberge Vie, $\mathrm{PhD}^{5}$, Linda Margareth Pedersen, $\mathrm{PhD}^{2}$, Kjersti Storheim, $\mathrm{PhD}^{2}$, Frank Skorpen, $\mathrm{PhD}^{6}$, Knut Hagen, $\mathrm{PhD}^{7,8}$, Johan Håkon Bjørngaard, PhD 5, 9, Bjørn Olav Åsvold, $\mathrm{PhD}^{3,10}$, John Anker Zwart, $\mathrm{PhD}^{1,2,4}$

m.b.johnsen@medisin.uio.no; bendik.s.winsvold@gmail.com; Sigrid.borte@gmail.com; gunnhild.vie@ntnu.no; limped@ous-hf.no; kjersti.storheim@medisin.uio.no;

frank.skorpen@ntnu.no; knut.hagen@ntnu.no; johan.h.bjorngaard@ntnu.no;

bjorn.o.asvold@ntnu.no; j.a.zwart@medisin.uio.no

${ }^{*}$ Corresponding author

Address: Research and Communication Unit for Musculoskeletal Health, Oslo University Hospital-Ullevål, Building 37B, PO Box 4956 Nydalen, N-0424 Norway Telephone no.: +4723016324 / Fax no.: +4723016150

Email: m.b.johnsen@medisin.uio.no

${ }^{1}$ Faculty of Medicine, Institute of Clinical Medicine, University of Oslo, Oslo, Norway

2 Research and Communication Unit for Musculoskeletal Health, Oslo University Hospital, Oslo, Norway

${ }^{3}$ K.G. Jebsen Center for Genetic Epidemiology, Department of Public Health and Nursing, NTNU, Norwegian University of Science and Technology, Trondheim, Norway

${ }^{4}$ Department of Neurology, Oslo University Hospital, Oslo, Norway 
${ }^{5}$ Department of Public Health and Nursing, NTNU, Norwegian University of Science and Technology, Trondheim, Norway

${ }^{6}$ Department of Clinical and Molecular Medicine, Faculty of Medicine and Health Sciences, NTNU, Norwegian University of Science and Technology, Trondheim, Norway

7 Department of Neuromedicine and Movement Science, NTNU, Norwegian University of Science and Technology, Trondheim, Norway

${ }^{8}$ Norwegian Advisory Unit on Headaches, St. Olavs Hospital, Trondheim University Hospital, Trondheim, Norway

${ }_{9}^{9}$ Forensic Department and Research Centre Brøset, St. Olavs Hospital, Trondheim University Hospital, Trondheim, Norway

${ }^{10}$ Department of Endocrinology, St. Olavs Hospital, Trondheim University Hospital, Trondheim, Norway

Total word count: 3496

Running title: The role of smoking on the risk of headache.

Keywords: headache, migraine, smoking, genetic variants, epidemiology. 


\section{ABSTRACT}

Background: Headache has been associated with various lifestyle- and psychosocial factors, one of which is smoking. The aim of the present study was to investigate whether the association between smoking intensity and headache is likely to be causal.

Method: 58316 participants from the Nord-Trøndelag Health Study (HUNT) with information on headache status were genotyped for the rs1051730 C > T single-nucleotide polymorphism (SNP). The SNP was used as an instrument for smoking intensity in a Mendelian randomization (MR) analysis. Association between rs1051730 T alleles and headache was estimated by odds ratios (OR) with 95\% confidence intervals (CI). Additionally, we investigated the association between the SNP and migraine or non-migrainous headache vs. no headache. All analyses were adjusted for age and sex.

Results: There was no strong evidence that the rs1051730 $\mathrm{T}$ allele was associated with headache in ever smokers (OR 0.99, 95\% CI 0.95-1.02). Similarly, there was no association between the rs1051730 T allele and migraine or non-migrainous headache vs. no headache.

Conclusion: Findings from this study do not support that there is a strong causal relationship between smoking intensity and any type of headache. Larger MR studies are required to examine whether higher smoking quantity can lead to a moderate increase in the risk of headache subtypes. 


\section{INTRODUCTION}

Primary headache disorders are highly prevalent, with migraine and tension-type headache being the most common, affecting $14.7 \%$ and $20.8 \%$ of the world's population respectively [1]. As much as $15 \%$ of the population report absence from work during the last year because of headache, and migraine alone is ranked as the $7^{\text {th }}$ leading cause of disability worldwide $[2,3]$. Even though headache has been linked to various lifestyle- and psychosocial factors [4-6], there is limited actionable knowledge about modifiable risk factors. Headache patients are often advised to change their lifestyle, including stopping smoking and increase their amount of physical exercise [5, 7]. However, the basis for such advice is largely based on epidemiological studies where definite conclusions about causal relationships cannot be drawn. Multiple studies have indicated that smoking is associated with headache with odds ratios from 1.3 to 1.5 [4, 5, 8]. The positive association seems to be true for both migraine and non-migrainous headache and is most evident among young adults [4]. Whether smoking causes headache or headache leads to altered smoking behavior is however uncertain.

Mendelian randomization (MR) offers an alternative approach to conventional observational studies, with the potential for stronger causal claims [9]. In MR, genetic variants are used as proxies for the exposure of interest. Since genetic variants are transmitted from parents to offspring at random during meiosis, they are not generally susceptible to confounding and reverse causation bias [9]. The single-nucleotide polymorphism (SNP) rs1051730 is the strongest genetic variant related to smoking behavior identified in genome-wide studies to date $[10,11]$. In smokers, each additional T allele of the rs1051730 SNP is associated with an increased intensity of smoking corresponding to around one additional cigarette per day [11, 12]. Our aim was to explore whether smoking intensity has a casual effect on headache, using a MR approach. 


\section{MATERIAL AND METHODS}

\section{Study population}

All inhabitants aged 20 years or older living in the county of Nord-Trøndelag, Norway, were invited to participate in the Nord-Trøndelag Health Study (HUNT) [13, 14]. For the present study, we included participants who had attended HUNT2 (1995-97) or HUNT3 (2006-08) (Figure 1 and Supplementary methods 1). All participants in HUNT have signed a written informed consent regarding the use of data from questionnaires and blood samples for research purposes $[13,14]$. The current study was approved by the Regional Committee for Medical and Health Research Ethics (REK), 2012/2291/REK South-East C.

\section{Genotyping}

The HUNT sample was genotyped using one of three different Illumina HumanCoreExome arrays (HumanCoreExome12 v1.0, HumanCoreExome12 v.1.1 and HumanCoreExome-24+ v1.1 with custom content). Imputation was performed using Minimac3 (v2.0.1, http://genome.sph.umich.edu/wiki/Minimac3) [15] with default settings and a customized Haplotype Reference consortium release 1.1 (HRC v1.1) [16]. (Supplementary methods 1).

\section{Smoking}

Smoking status was self-reported and categorised as never, former and current daily smokers. Current smokers were asked to report the average number of cigarettes smoked per day (Supplementary methods 1).

\section{Outcome}

In the HUNT questionnaire, those who answered "yes" to "Have you suffered from headache during the last 12 months?" were classified as headache sufferers. Those who answered "no" were defined as headache-free controls. The headache sufferers were further classified as having 
migraine or non-migrainous headache, based on a modified version of the International Classification of Headache Disorders (ICHD II) [17] (Supplementary methods 1).

\section{Statistical methods}

Descriptive statistics according to the number of rs1051730 T alleles were compared using $\chi^{2}$ test for categorical variables and a linear regression for continuous variables. We assumed an additive genetic model, so risk estimates represent the odds ratios (ORs) per additional copy of the $\mathrm{T}$ allele.

\section{SNP-exposure association:}

We used linear regression to estimate the association between rs1051730 $\mathrm{T}$ alleles and smoking intensity.

\section{Exposure-outcome association (observational analysis):}

Logistic regression with ORs and 95\% confidence intervals (CIs) was used to estimate the relationship between smoking intensity, or smoking status, and headache (Supplementary methods 1). In a sensitivity analysis, we restricted former smokers to those who reported smoking cessation $>1$ year ago to avoid bias from current smoking at time of reporting headache.

\section{SNP-outcome association (MR analysis):}

Logistic regression was used to estimate the relationship between rs1051730 $\mathrm{T}$ alleles and headache (Supplementary methods 1). The main analysis was in strata of never vs. ever smokers (current and former smokers combined). Additionally, we stratified the SNP-headache association by current, former or never smoking. In a secondary analysis, we investigated the association between the SNP and migraine, or non-migrainous headache, vs. no headache, using a multinomial logistic regression. We also performed the MR analysis stratified by age $<$ vs. $\geq 40$ 
[4]. All analyses were adjusted for age and sex. Additionally, we adjusted for BMI as a potential confounder/mediator.

\section{RESULTS}

In total 58316 participants were genotyped for rs1051730 and had information about headache. Of these, 25666 (46.0\%) were never smokers, 14799 (26.5\%) former smokers, 15 $278(27.4 \%)$ current smokers (Table 1). Among the 58316 participants, 39.4\% reported headache; $12.9 \%$ had migraine and $26.5 \%$ had non-migrainous headache (Figure 1 ). The probability of being a current smoker and the number of cigarettes smoked per day increased by number of rs1051730 $\mathrm{T}$ alleles. There was a slight decrease in age and BMI according to increasing number of $\mathrm{T}$ alleles. There was no difference in the prevalence of headache according to the number of $\mathrm{T}$ alleles (Table 1 ).

\section{SNP-exposure association:}

Among current smokers, each additional T allele was associated with 0.68, 95\% CI 0.55-0.80 additional cigarettes smoked per day.

\section{Observational analysis:}

There was a positive association between smoking intensity and headache (Figure 2). The association (OR) between each additional cigarette smoked per day and headache was 1.02 (95\% CI 1.01-1.02). According to smoking status, both current smoking (OR 1.30, 95\% CI 1.251.36) and former smoking (OR 1.24, 95\% CI 1.19-1.30), compared to never smoking, were positively associated with headache. Former smokers (cessation > 1 year ago) compared with never smokers also had increased odds of headache, OR 1.24 (95\% CI 1.18-1.29).

MR analysis:

There was no association between rs1051730 T alleles and headache in ever smokers (OR 0.99, 95\% CI 0.95-1.02) or in never smokers (OR 1.00, 95\% CI 0.96-1.04) (Table 2). Moreover, there 
was no association between the rs1051730 T allele and headache in current smokers (OR 1.00, 95\% CI 0.96-1.06) (Table 3).

We found no association between the $\mathrm{T}$ allele and migraine or non-migrainous headache vs no headache (Table 2 and Table 3 ). Stratified analysis by age ( $<40$ or $\geq 40$ years) showed no statistically significant associations between the rs1051730 $\mathrm{T}$ allele and headache vs no headache (Supplementary Table 1). Adjustments for BMI did not change the association between the $\mathrm{T}$ allele and any type of headache (data not shown).

\section{DISCUSSION}

We did not find evidence for a causal effect of smoking intensity on headache. While a positive association was observed between the number of cigarettes smoked per day and headache, there was no association between the rs1051730 T alleles and any type of headache. The results indicate that there is no strong causal effect of smoking intensity on headache overall.

The observational association between smoking status and headache in the present study is comparable to results from previous studies (ORs 1.3 to 1.6) $[4,5,8]$. Regarding smoking intensity, Gan et al. [18] found in a cross-sectional survey that the number of cigarettes smoked per day was associated with headache. Smoking between 11-18 cigarettes per day was associated with an OR of 1.53 for having headache, compared to never smokers [18]. In the observational analysis, we found the OR for headache to be 1.02 per cigarette, corresponding to an OR between 1.20-1.50 for headache if you smoked 10-20 cigarettes per day. The association between former smoking and headache was also present for former smokers who had quit smoking >1 year ago (OR 1.24), which confirmed that the observed association was not due to current smoking at time of reporting headache alone. However, the magnitude of the observational effect estimate should not be given too much emphasis. The current study was designed for MR analysis, where the aim was to determine whether the association observed between smoking and headache is likely to be causal, and not for determining the exact magnitude of the effect estimate. The significant association we found in the observational 
analysis was, however, not replicated in the MR analysis. Still, we cannot refute by our MR analysis that a moderate causal association of smoking intensity on headache exists, as the $95 \%$ CI for our MR analysis included the magnitude of the observational association.

A former study on the same HUNT population found an association between current smoking (for those who smoked $\geq 10$ cigarettes per day) and headache, in those younger than 40 years of age (OR 1.50), but not in those age $\geq 40$ years [4]. However, the headache diagnoses in HUNT include only current headache (present within the last 12 months), which might partly explain this finding, since headache prevalence, and in particular migraine prevalence, is decreasing in the older age groups [19]. We therefore stratified by age 40 to investigate whether a causal effect of smoking might depend on age. Although the point estimate for the association was higher for those aged $<40$ (OR 1.07) compared to those aged $\geq 40$ years (OR 0.97), there was no significant association in either group.

The large sample size and the use of a well-defined genetic instrument are strengths of this study. Using rs1051730 as a proxy for smoking intensity allowed us to examine the relationship between smoking and headache in a pseudo-randomized manner, avoiding confounding and reversed causation. Another strength is the use of validated headache diagnoses, but the use of questionnaire-based diagnoses will necessarily give some degree of misclassification, which could deflate our estimates. This deflation would probably have been most pronounced for non-migrainous headache, which had the lowest agreement between questionnaire-based and interview diagnoses [20,21]. Another limitation is that we assessed current headache only, causing headache-sufferers who have become headache-free prior to baseline to be classified as controls. Such misclassification is likely to weaken any observed effect, in particular, in the older age groups where cessation rates are high [19].

Non-participation in HUNT has been associated with an unhealthier lifestyle with respect to tobacco smoking, physical inactivity and poor somatic health status [22]. Non-responders to the headache question in the current study were more often men, current smokers and younger 
(data not shown). This is in line with the overall characteristics of non-responders to the headache questions in HUNT [23]. While we cannot rule out an effect of selection bias, it is unlikely that the association between rs1051730 and headache should be substantially different among participants versus non-participants.

MR studies rely on three main assumptions: 1) The genetic variant is associated with the exposure, 2) the genetic variant is associated with the outcome through the studied exposure only, and 3) the genetic variant is independent of other factors that affect the outcome [9]. Regarding assumption 1, the association between rs1051730 and smoking intensity has been confirmed in previous genome-wide association studies $[10,11]$ and the association was verified in the current data. The second assumption is violated if the genetic variant has multiple effects, or if the genetic variant is in linkage disequilibrium with another variant that affects the outcome in other ways than through the exposure of interest. If we found an association between the rs1051730 $\mathrm{T}$ allele and headache in never smokers, we might be suspicious of such a violation since the SNP is a proxy for smoking intensity. This can be considered a test of the second assumptions; that the genetic variant only operates on the outcome via its effect on the exposure (i.e. no pleiotropy) [9]. However, in our case, no association was seen in any of the strata of smoking status and the results did not change after adjustments for BMI.

Stratification of a MR analysis by smoking status groups may lead to collider bias by conditioning on a common observed measure [9], thus potentially introduce bias from confounders to the exposure-outcome association that may violate the third assumption. For this reason, we performed the MR analysis in broader strata of never vs ever smokers, and adjusted for BMI, but no association between the rs1051730 $\mathrm{T}$ alleles and headache was seen.

To conclude, this MR analysis suggests that there is no strong causal effect of smoking intensity on headache. However, larger MR studies are required to examine whether higher smoking quantity can lead to a moderate increase in the risk of headache subtypes. 


\section{Acknowledgements}

The Nord-Trøndelag Health Study (The HUNT Study) is a collaboration between the HUNT Research Centre, Faculty of Medicine and Health Sciences, the Norwegian University of Science and Technology (NTNU), Nord-Trøndelag County Council, Central Norway Regional Health Authority and the Norwegian Institute of Public Health.

\section{Author contributions}

All authors contributed to the conception and design of the study. MBJ, BSW, KS and JAZ were responsible for acquisition of the data. MBJ and GÅV performed the analyses of the data, while all authors contributed to the interpretation of data. MBJ, BSW and SB drafted the first version of the manuscript. All authors revised the manuscript and gave their final approval of the submitted version.

\section{Funding}

MBJ and GÅV are supported by the Research Council of Norway (RCN; grant number 248817 and 250335). National Institute of Health (NIH), University of Michigan, RCN, Central Norway Regional Health Authority, Faculty of Medicine, the Norwegian University of Science and Technology (NTNU) and the K. G. Jebsen Center for Genetic Epidemiology financed the genotyping. The funding source had no involvement in any aspect of this manuscript.

\section{Disclosure of conflict of interest}

None declared. 


\section{Table captions}

Table 1 Baseline characteristics of the study participants.

Table 2 Association between rs1051730 T alleles and headache.

Table 3 Association between rs1051730 T alleles and headache according to smoking status. Supplementary methods 1 Additional description of material and methods.

Supplementary Table 1 Association between rs1051730 T alleles and headache according to age.

\section{Figure captions}

Figure 1 Flow chart of the study sample from the Nord-Trøndelag Health Study (HUNT).

Figure 2 Age- and sex-adjusted ORs for headache with 95\% CI (grey) according to numbers of cigarettes smoked per day. Never and former smokers (zero cigarettes a day) are used as a reference group. 


\section{References}

1. Global, regional, and national incidence, prevalence, and years lived with disability for 301 acute and chronic diseases and injuries in 188 countries, 1990-2013: a systematic analysis for the Global Burden of Disease Study 2013. Lancet. 2015;386(9995):743-800.

2. Stovner LJ, Zwart JA, Hagen K, Terwindt GM, Pascual J. Epidemiology of headache in Europe. Eur J Neurol. 2006;13(4):333-45.

3. Vos T, Allen C, Arora M, Barber RM, Bhutta ZA, Brown A, et al. Global, regional, and national incidence, prevalence, and years lived with disability for 310 diseases and injuries, 1990-2015: a systematic analysis for the Global Burden of Disease Study 2015. Lancet. 2016;388(10053):1545-602.

4. Aamodt AH, Stovner LJ, Hagen K, Brathen G, Zwart J. Headache prevalence related to smoking and alcohol use. The Head-HUNT Study. Eur J Neurol. 2006;13(11):1233-8.

5. Robberstad L, Dyb G, Hagen K, Stovner LJ, Holmen TL, Zwart JA. An unfavorable lifestyle and recurrent headaches among adolescents: the HUNT study. Neurology. 2010;75(8):712-7.

6. Dyb G, Stensland S, Zwart JA. Psychiatric comorbidity in childhood and adolescence headache. Current pain and headache reports. 2015;19(3):5.

7. Penzien DB, Rains JC, Lipchik GL, Creer TL. Behavioral interventions for tension-type headache: overview of current therapies and recommendation for a self-management model for chronic headache. Current pain and headache reports. 2004;8(6):489-99.

8. Molarius A, Tegelberg A, Ohrvik J. Socio-economic factors, lifestyle, and headache disorders - a population-based study in Sweden. Headache. 2008;48(10):1426-37.

9. Haycock PC, Burgess S, Wade KH, Bowden J, Relton C, Davey Smith G. Best (but oftforgotten) practices: the design, analysis, and interpretation of Mendelian randomization studies. Am J Clin Nutr. 2016. 
10. Liu JZ, Tozzi F, Waterworth DM, Pillai SG, Muglia P, Middleton L, et al. Meta-analysis and imputation refines the association of 15q25 with smoking quantity. Nat Genet. 2010;42(5):43640.

11. Thorgeirsson TE, Geller F, Sulem P, Rafnar T, Wiste A, Magnusson KP, et al. A variant associated with nicotine dependence, lung cancer and peripheral arterial disease. Nature. 2008;452(7187):638-42.

12. Munafo MR, Timofeeva MN, Morris RW, Prieto-Merino D, Sattar N, Brennan P, et al. Association between genetic variants on chromosome 15q25 locus and objective measures of tobacco exposure. J Natl Cancer Inst. 2012;104(10):740-8.

13. Holmen J, Midthjell K, Krüger Ø, Langhammer A, Holmen TL, Bratberg G, et al. The NordTrøndelag Health Study 1995-97 (HUNT 2): Objectives, contents, methods and participation. Norsk Epidemiologi. 2003;13(1):19-32.

14. Krokstad S, Langhammer A, Hveem K, Holmen TL, Midthjell K, Stene TR, et al. Cohort Profile: the HUNT Study, Norway. Int J Epidemiol. 2013;42(4):968-77.

15. Das S, Forer L, Schonherr S, Sidore C, Locke AE, Kwong A, et al. Next-generation genotype imputation service and methods. Nat Genet. 2016;48(10):1284-7.

16. McCarthy S, Das S, Kretzschmar W, Delaneau O, Wood AR, Teumer A, et al. A reference panel of 64,976 haplotypes for genotype imputation. Nat Genet. 2016;48(10):1279-83.

17. The International Classification of Headache Disorders: 2nd edition. Cephalalgia. 2004;24 Suppl 1:9-160.

18. Gan WQ, Estus S, Smith JH. Association Between Overall and Mentholated Cigarette Smoking With Headache in a Nationally Representative Sample. Headache. 2016;56(3):511-8. 19. Stovner LJ. Headache epidemiology: how and why? J Headache Pain. 2006;7(3):141-4. 20. Hagen K, Zwart JA, Aamodt AH, Nilsen KB, Brathen G, Helde G, et al. The validity of questionnaire-based diagnoses: the third Nord-Trondelag Health Study 2006-2008. J Headache Pain. 2010;11(1):67-73. 
21. Hagen K, Zwart JA, Vatten L, Stovner LJ, Bovim G. Head-HUNT: validity and reliability of a headache questionnaire in a large population-based study in Norway. Cephalalgia : an international journal of headache. 2000;20(4):244-51.

22. Langhammer A, Krokstad S, Romundstad P, Heggland J, Holmen J. The HUNT study: participation is associated with survival and depends on socioeconomic status, diseases and symptoms. BMC Med Res Methodol. 2012;12:143.

23. Linde M, Stovner LJ, Zwart JA, Hagen K. Time trends in the prevalence of headache disorders. The Nord-Trondelag Health Studies (HUNT 2 and HUNT 3). Cephalalgia. 2011;31(5):585-96. 


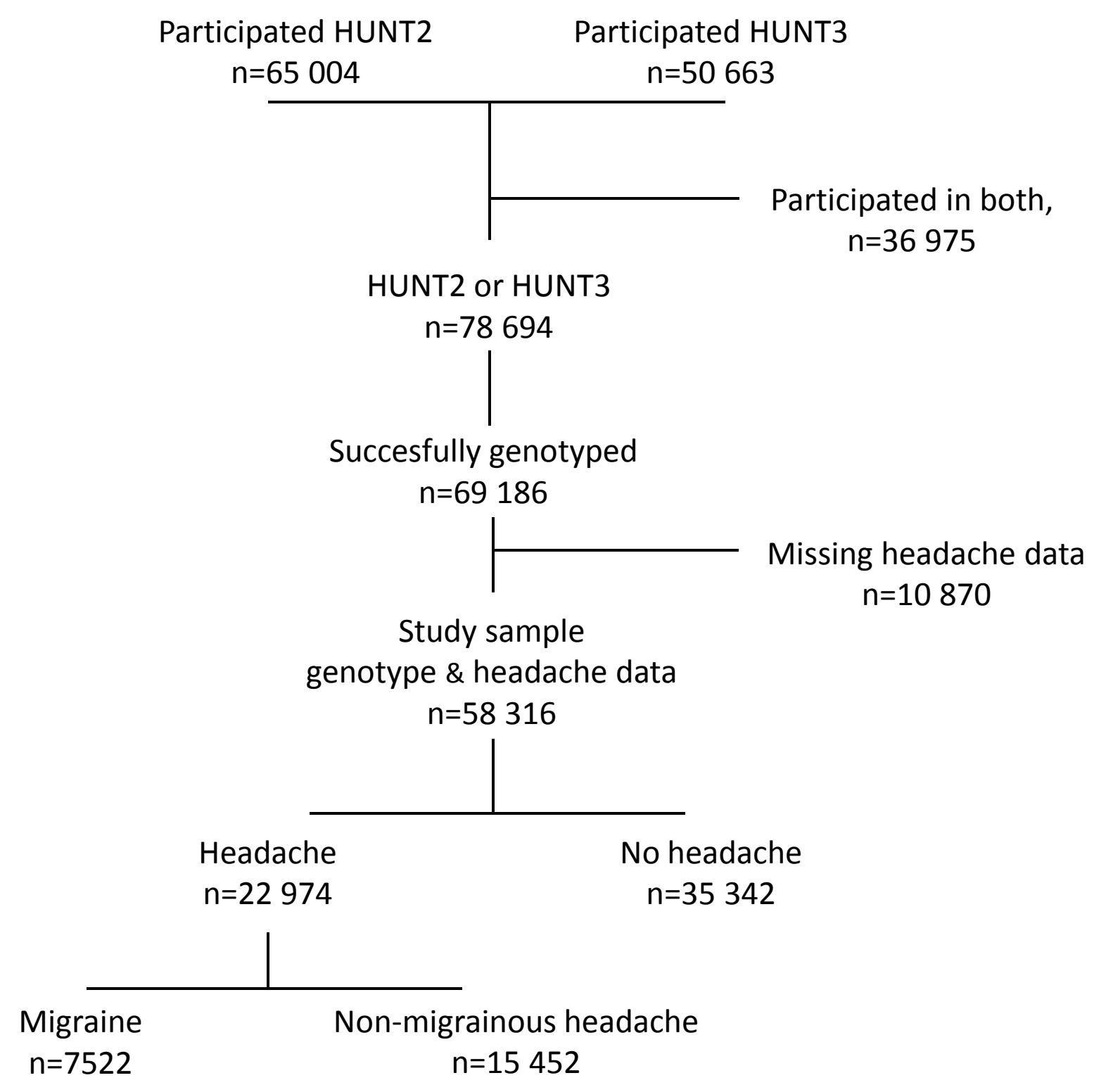




\section{Supplementary material 1 Additional description of the material and methods.}

\section{Study population}

All inhabitants aged 20 years or older living in the county of Nord-Trøndelag, Norway, were invited to participate in the Nord-Trøndelag Health Study (HUNT). For the present study, we included participants who had attended HUNT2 (1995-97) or HUNT3 (2006-08) [1, 2]. We used HUNT2 as our main cohort and additionally included participants from HUNT3 who had not attended HUNT2 (Figure 1). This resulted in 78694 participants, of which 69186 had been successfully genotyped. After excluding 10870 with missing headache data, our study sample comprised 58316 participants.

\section{Genotyping}

DNA was extracted from blood samples collected at baseline in either HUNT2 or HUNT3 and stored at the HUNT Biobank. Genotyping was performed using one of three different Illumina HumanCoreExome arrays (HumanCoreExome12 v1.0, HumanCoreExome12 v.1.1 and HumanCoreExome-24+ v1.1 with custom content). Samples with call rate $<99 \%$, or that failed other quality control criteria were excluded. Imputation was performed using Minimac3 (v2.0.1, http://genome.sph.umich.edu/wiki/Minimac3) [3] with default settings and a customized Haplotype Reference consortium release 1.1 (HRC v1.1) [4]. Imputed variants with $\mathrm{R}^{2}<0.3$ or estimated minor allele frequency (MAF) below $0.05 \%$ were excluded. Imputation quality for the rs1051730 SNP was high with $\mathrm{R}^{2}=0.9999$ and MAF=0.335, which was in agreement to HapMapCEU data (MAF=0.389). There was no evidence of departure from the Hardy-Weinberg equilibrium $\left(\chi^{2}\right.$ test, $\left.\mathrm{p}=0.20\right)$. 


\section{Smoking}

Smoking status was self-reported in the questionnaire, categorised as never, former and current daily smokers. Current smokers were asked to report the average number of cigarettes smoked per day and former smokers to report the time since smoking cessation. Information about occasional smoking was only available from HUNT3. To obtain a homogenous exposure group of current daily smokers, we excluded those who reported to be occasional smokers only ( $\mathrm{n}=1194$ from HUNT3) from the analyses stratified by current, former and never smoking (presented in Table 3).

\section{Outcome}

In the questionnaire, those who answered "yes" to "Have you suffered from headache during the last 12 months?" were classified as headache sufferers. Those who answered "no" were defined as headache-free controls. The headache sufferers were further classified as having migraine or non-migrainous headache, based on a modified version of the International Classification of Headache Disorders (ICHD II) [5]. Based on the subsequent headache questions [6, 7], headache sufferers were classified as having migraine if they fulfilled all of the following criteria: (1) headache lasting from 4-72 hours (4 hours were accepted for those who reported commonly occurring visual disturbances before headache); (2) headache with at least one of the following characteristics: pulsating quality, unilateral location or aggravation by physical activity; (3) during headache, at least one of the following occurred: nausea, photophobia and phonophobia. In addition, the participants were asked if they suffered from migraine; those who responded positively to this question were also included in the migraine group. Headache sufferers who did not fulfill the criteria for migraine were classified as having non-migrainous headache. The headache diagnoses have previously been validated by clinical interviews performed by neurologists $[6,7]$, where the highest sensitivity and specificity were reported for headache (between $85-88 \%$ and $83-86 \%$, respectively), then migraine (between $49-69 \%$ and $89-96 \%$, 
respectively) and lowest for non-migrainous headache (between $56-61 \%$ and $77-81 \%$, respectively) [6, 7]. The main outcome in the MR analysis was headache vs no headache.

\section{Statistical methods}

\section{Exposure-outcome association (observational analysis):}

Logistic regression with ORs and 95\% confidence intervals (CIs) was used to estimate the relationship between smoking intensity, or smoking status, and headache. To illustrate the association between the number of cigarettes smoked and the risk of headache, smoking intensity was expressed by a restricted cubic spline, which is a flexible description of a nonlinear relationship, with knots at 0,10, 20 and 30 cigarettes per day. Never and former smokers were assumed to smoke zero cigarettes and were used as the reference point. In a sensitivity analysis, we restricted former smokers to those who reported smoking cessation $>1$ year ago to avoid bias from current smoking at time of reporting headache.

\section{SNP-outcome association (MR analysis):}

We performed the MR analysis, using logistic regression to estimate the relationship between rs1051730 T alleles and headache. Carriers of the rs1051730 T alleles may be less likely to quit smoking. Thus, stratification on current smoking may lead to collider bias by conditioning on an observed measure of exposure [8]. Therefore, the main analysis was in strata of never vs. ever smokers (current and former smokers combined). Additionally, we stratified the SNP-headache association by current, former or never smoking. In a secondary analysis, we investigated the association between the SNP and migraine, or non-migrainous headache, vs. no headache, using a multinomial logistic regression. We also performed the main MR analysis stratified by age < vs. $\geq 40$, based on previous results of an interaction between smoking and age [4]. All analyses were adjusted for age and sex. 


\section{References}

1. Holmen J, Midthjell K, Krüger $\emptyset$, Langhammer A, Holmen TL, Bratberg G, et al. The NordTrøndelag Health Study 1995-97 (HUNT 2): Objectives, contents, methods and participation. Norsk Epidemiologi. 2003;13(1):19-32.

2. Krokstad S, Langhammer A, Hveem K, Holmen TL, Midthjell K, Stene TR, et al. Cohort Profile: the HUNT Study, Norway. Int J Epidemiol. 2013;42(4):968-77.

3. Das S, Forer L, Schonherr S, Sidore C, Locke AE, Kwong A, et al. Next-generation genotype imputation service and methods. Nat Genet. 2016;48(10):1284-7.

4. McCarthy S, Das S, Kretzschmar W, Delaneau O, Wood AR, Teumer A, et al. A reference panel of 64,976 haplotypes for genotype imputation. Nat Genet. 2016;48(10):1279-83.

5. The International Classification of Headache Disorders: 2nd edition. Cephalalgia. 2004;24 Suppl 1:9-160.

6. Hagen K, Zwart JA, Aamodt AH, Nilsen KB, Brathen G, Helde G, et al. The validity of questionnairebased diagnoses: the third Nord-Trondelag Health Study 2006-2008. J Headache Pain. 2010;11(1):67-73. 7. Hagen K, Zwart JA, Vatten L, Stovner LJ, Bovim G. Head-HUNT: validity and reliability of a headache questionnaire in a large population-based study in Norway. Cephalalgia : an international journal of headache. $2000 ; 20(4): 244-51$.

8. Haycock PC, Burgess S, Wade KH, Bowden J, Relton C, Davey Smith G. Best (but oft-forgotten) practices: the design, analysis, and interpretation of Mendelian randomization studies. Am J Clin Nutr. 2016. 
Table 1. Baseline characteristics of the study participants.

\begin{tabular}{|c|c|c|c|c|c|}
\hline & \multirow[b]{2}{*}{$\mathrm{n}^{\mathrm{a}}$} & \multicolumn{3}{|c|}{ No. of rs 1051730 T alleles } & \multirow[b]{2}{*}{$p$-value ${ }^{b}$} \\
\hline & & 0 & 1 & 2 & \\
\hline Study sample* - \% & 58316 & 44.4 & 44.3 & 11.3 & \\
\hline \multicolumn{6}{|l|}{ Smoking - \% } \\
\hline Never & 25666 & 46.4 & 45.7 & 45.6 & \\
\hline Former & 14799 & 27.2 & 26.2 & 25.1 & \\
\hline Current daily & 15287 & 26.3 & 28.1 & 29.3 & $<0.001$ \\
\hline No. of cigarettes per day ${ }^{c}-$ mean & 15003 & 10.7 & 11.4 & 12.1 & 0.008 \\
\hline Headache - \% & 22974 & 39.3 & 39.4 & 39.5 & 0.96 \\
\hline Migraine & 7522 & 12.9 & 13.0 & 12.7 & \\
\hline Non-migrainous headache & 15452 & 26.4 & 26.5 & 26.8 & 0.96 \\
\hline Age (years) - mean & 58313 & 48.1 & 47.9 & 47.6 & 0.03 \\
\hline BMI $\left(\mathrm{kg} / \mathrm{m}^{2}\right)-$ mean & 58106 & 26.4 & 26.3 & 26.2 & 0.001 \\
\hline Women-\% & 31446 & 53.5 & 54.2 & 54.8 & 0.08 \\
\hline \multicolumn{6}{|l|}{ Physical activity ${ }^{d}-\%$} \\
\hline Low & 31948 & 57.4 & 57.6 & 58.6 & \\
\hline High & 23509 & 42.6 & 42.4 & 41.4 & 0.21 \\
\hline
\end{tabular}

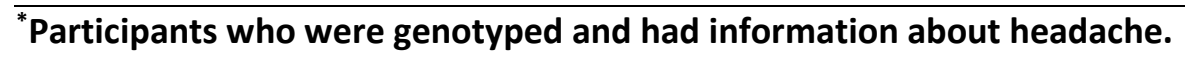

${ }^{\text {aTotal }} \mathrm{n}$ varies from 55457 to 58316 due to missing data.

${ }^{b} \chi^{2}$ test for categorical variables and linear regression for linear associations according to number of T alleles.

${ }^{\mathrm{c}}$ Among current smokers.

$\mathrm{BMI}=$ body mass index .

${ }^{\mathrm{d} B a s e d}$ on a weekly average of duration and intensity of physical activities during the last year; low=not being sweat or out of breath, high=being sweat or out of breath. 


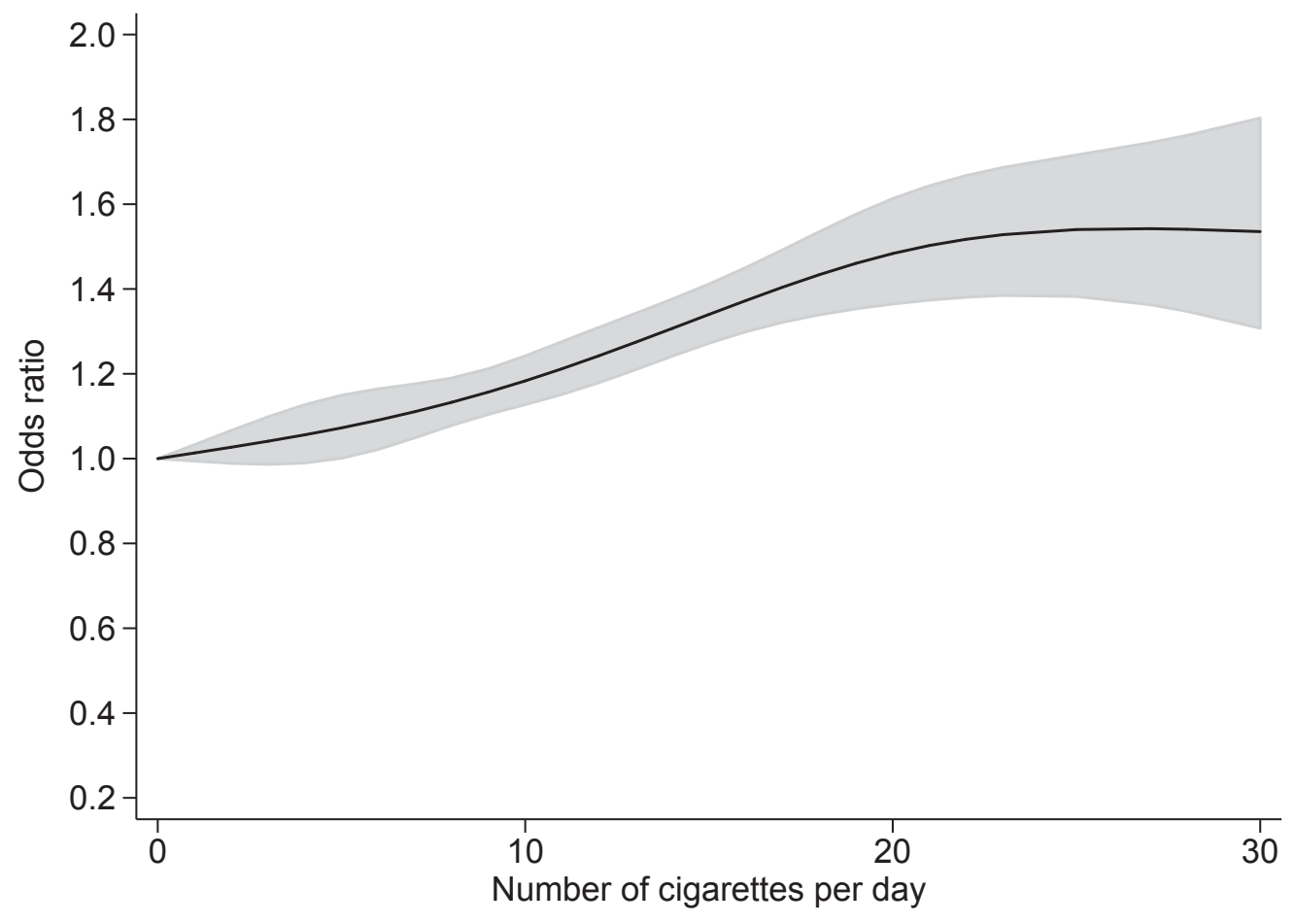


Table 2. Association between rs1051730 T alleles and headache.

\begin{tabular}{cccccccc}
\hline & All participants & & Never smokers & & Ever smokers \\
\multicolumn{1}{c}{ Per T allele effect } & OR $(95 \% \mathrm{Cl})$ & p-value & OR $(95 \% \mathrm{Cl})$ & $\mathrm{p}$-value & OR $(95 \% \mathrm{Cl})$ & $\mathrm{p}$-value \\
\hline Main analysis $^{\mathrm{a}}$ & & & & & & \\
Headache & $0.99(0.97-1.02)$ & 0.61 & $1.00(0.96-1.04)$ & 0.91 & $0.99(0.95-1.02)$ & 0.41 \\
Secondary analysis & & & & & & & \\
Migraine & $0.98(0.95-1.02)$ & 0.38 & $0.99(0.93-1.04)$ & 0.63 & $0.97(0.92-1.03)$ & 0.32 \\
Non-migrainous headache & $1.00(0.97-1.03)$ & 0.89 & $1.00(0.96-1.05)$ & 0.88 & $0.99(0.95-1.03)$ & 0.63 \\
\hline
\end{tabular}

OR=odds ratio, $\mathrm{Cl}=$ confidence interval.

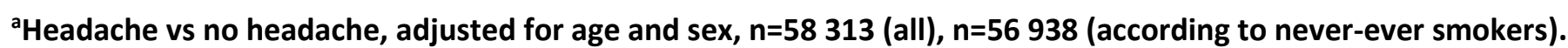

${ }^{b}$ Migraine or non-migrainous headache vs no headache, adjusted for age and sex, $n=58313$ (all), n=56 938 (according to never-ever smokers). 
Table 3. Association between rs1051730 T alleles and headache according to smoking status.

\begin{tabular}{|c|c|c|c|c|c|c|}
\hline & Never smokers & & Former smokers & & Current smokers & \\
\hline Per T allele effect & OR $(95 \% \mathrm{Cl})$ & $p$-value & OR $(95 \% \mathrm{Cl})$ & $p$-value & OR $(95 \% \mathrm{Cl})$ & $\mathrm{p}$-value \\
\hline \multicolumn{7}{|l|}{ Main analysis ${ }^{a}$} \\
\hline Headache & $1.00(0.96-1.04)$ & 0.89 & $0.97(0.92-1.02)$ & 0.24 & $1.00(0.96-1.06)$ & 0.87 \\
\hline \multicolumn{7}{|l|}{ Secondary analysis ${ }^{b}$} \\
\hline Migraine & $0.99(0.93-1.04)$ & 0.62 & $1.01(0.93-1.09)$ & 0.82 & $0.95(0.89-1.03)$ & 0.21 \\
\hline $\begin{array}{r}\text { Non-migrainous } \\
\text { headache }\end{array}$ & $1.00(0.96-1.05)$ & 0.89 & $0.95(0.90-1.01)$ & 0.10 & $1.03(0.97-1.08)$ & 0.35 \\
\hline
\end{tabular}

$\mathrm{OR}=$ odds ratio, $\mathrm{Cl}=$ confidence interval.

${ }^{a}$ Headache vs no headache, adjusted for age and sex, $n=55749$ (according to smoking status).

${ }^{b}$ Migraine or non-migrainous headache vs no headache, adjusted for age and sex, $n=55749$ (according to smoking status). 